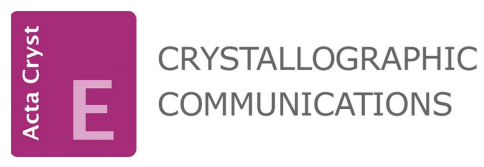

ISSN 2056-9890

Received 22 January 2020

Accepted 13 February 2020

Edited by M. Weil, Vienna University of Technology, Austria

Keywords: crystal structure; sulfonamide; $\pi-\pi$ interaction; $\mathrm{C}-\mathrm{H}$... O hydrogen bonds; $\mathrm{C}-$ $\mathrm{H} \cdots \pi$ interactions; polymorphism.

CCDC reference: 1983920

Supporting information: this article has supporting information at journals.iucr.org/e
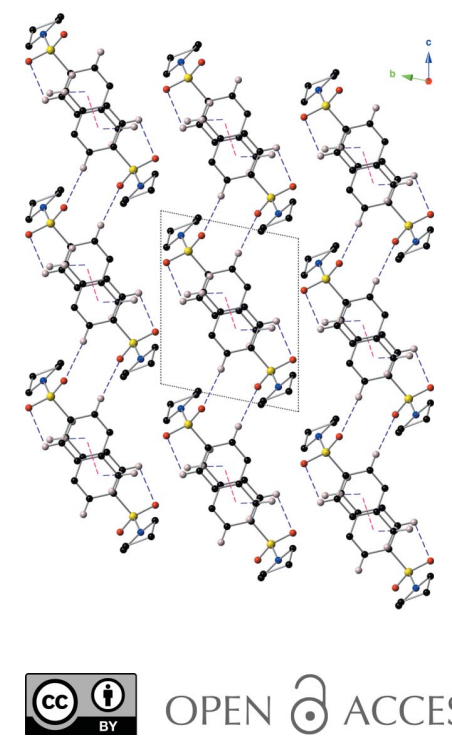

\title{
Crystal structure of 1-[(4-methylbenzene)sulfonyl]- pyrrolidine
}

\author{
Brock A. Stenfors, ${ }^{a}$ Richard J. Staples, ${ }^{b}$ Shannon M. Biros ${ }^{a}$ and Felix N. Ngassa ${ }^{a *}$ \\ ${ }^{\mathbf{a}}$ Department of Chemistry, 1 Campus Dr., Grand Valley State University, Allendale, MI 49401, USA, and ${ }^{\mathbf{b}}$ Center for \\ Crystallographic Research, Michigan State University, Department of Chemistry and Chemical Biology, East Lansing, MI \\ 48824, USA. *Correspondence e-mail: ngassaf@gvsu.edu
}

The molecular structure of the title compound, $\mathrm{C}_{11} \mathrm{H}_{15} \mathrm{NO}_{2} \mathrm{~S}$, features a sulfonamide group with $\mathrm{S}=\mathrm{O}$ bond lengths of 1.4357 (16) and 1.4349 (16) $\AA$, an $\mathrm{S}-\mathrm{N}$ bond length of 1.625 (2) $\AA$, and an $\mathrm{S}-\mathrm{C}$ bond length of 1.770 (2) $\mathrm{A}$. When viewing the molecule down the $\mathrm{S}-\mathrm{N}$ bond, both $\mathrm{N}-\mathrm{C}$ bonds of the pyrrolidine ring are oriented gauche to the $\mathrm{S}-\mathrm{C}$ bond with torsion angles of $-65.6(2)^{\circ}$ and $76.2(2)^{\circ}$. The crystal structure features both intra- and intermolecular $\mathrm{C}-\mathrm{H}$... O hydrogen bonds, as well as intermolecular $\mathrm{C}-$ $\mathrm{H} \cdots \pi$ and $\pi-\pi$ interactions, leading to the formation of sheets parallel to the $a c$ plane.

\section{Chemical context}

Sulfonamides are of significant value in organic chemistry because of their therapeutic properties. These molecules are referred to in the pharmaceutical industry as sulfa drugs. This class of drugs has been widely used in various pharmaceutical applications owing to their antibacterial, antiviral, antimalarial, antifungal, anticancer, antidepressant, and other properties (Apaydın \& Török, 2019).

$\mathrm{N}$-containing heterocycles have found many uses in pharmaceutical and materials sciences, and as a result they have attracted the attention of many in the synthetic community. Numerous synthetic methods leading to N-containing heterocycles have been reported (Jiang \& Ma, 2013). Notwithstanding, because of the importance of N-containing heterocycles, new and versatile synthetic methods are still desirable. The pyrrolidine-4-methylbenzenesulfonamide moiety is found in a variety of biologically important compounds that exhibit anti-inflammatory properties. Lproline-derived 4-methylbenzenesulfonamides (Fig. 1) have been reported to exhibit anti-inflammatory activity against Trypanosoma brucei gambiense (Ugwu et al., 2018). Furthermore, these compounds can permeate the blood-brain barrier and hence can be used in treating inflammation of the brain (Ugwu et al., 2017).

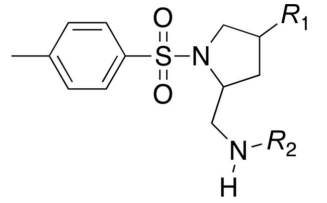

Figure 1

L-proline-derived 4-methylbenzenesulfonamide compounds that have been reported to exhibit anti-inflammatory activity against (a) Trypanosoma brucei gambiense and (b) to reduce brain inflammation. 
Generally, sulfonamides are synthesized by an analogous nucleophilic acyl-substitution reaction between an electrophile and a nucleophilic amine (Patel et al., 2018). Efficient methods from the literature involve the base-catalyzed sulfonylation of amines using sulfonyl halides (Yan et al., 2007) or sulfonic acids (De Luca \& Giacomelli, 2008) as electrophiles. The title compound, along with some related analogs, has been synthesized previously (Ohwada, et al., 1998). Recently, we have discovered a more efficient method using aqueous potassium carbonate as the base. This method avoids the use of a phase-transfer catalyst by using tetrahydrofuran as a water-miscible solvent. An increased rate of reaction and yield of sulfonamide compounds produced from a wide range of amines has been observed. These reaction conditions produced the title compound in a $91 \%$ yield, compared to the $58 \%$ yield previously reported.

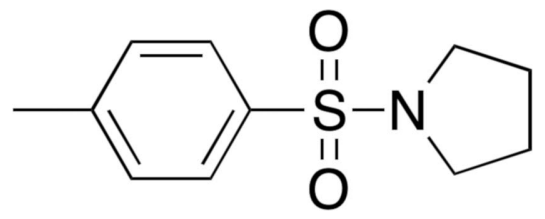

In a continuation of our research group's ongoing interest in synthesizing small sulfonamide molecules that mimic the structural motifs of known sulfonamide drug candidates, we synthesized the title compound, $\mathrm{C}_{11} \mathrm{H}_{15} \mathrm{NO}_{2} \mathrm{~S}$, and determined its crystal structure from single-crystal $\mathrm{X}$-ray diffraction data.

\section{Structural commentary}

The molecular structure of the title compound is shown in Fig. 2. The $\mathrm{S} 1=\mathrm{O} 1$ and $\mathrm{S} 1=\mathrm{O} 2$ bond lengths are 1.4357 (16) and 1.4349 (16) $\AA$, which is in line with known values. The $\mathrm{S} 1-\mathrm{C} 5$ and $\mathrm{S} 1-\mathrm{N} 1$ bond lengths are 1.770 (2) and 1.625 (2) $\AA$, respectively, with an N1-S1 - C5 bond angle of $107.66(9)^{\circ}$. The $\tau_{4}$ descriptor for fourfold coordination around the sulfur atom, S1, is 0.94, indicating a slightly distorted tetrahedron (ideal values are 0 for square-planar, 0.85 for trigonal-pyramidal, and 1 for tetrahedral coordination; Yang et al., 2007). Both $\mathrm{C}-\mathrm{N}$ bonds of the pyrrolidine

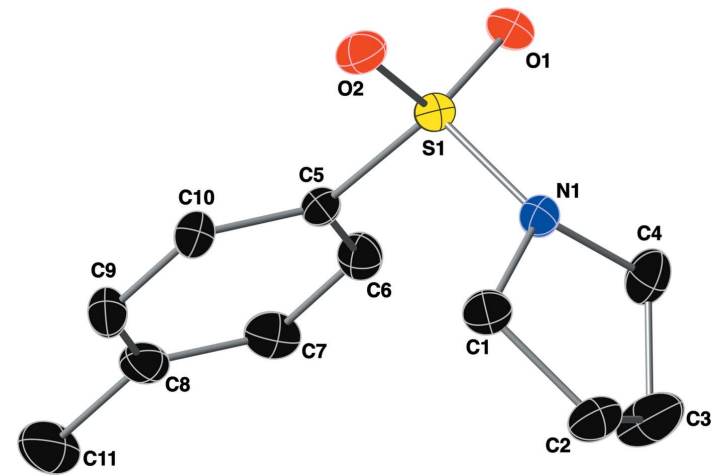

Figure 2

The molecular structure of the title compound, with the atom-labeling scheme. Displacement ellipsoids are drawn at the $40 \%$ probability level, and all hydrogen atoms have been omitted for clarity.
Table 1

Hydrogen-bond geometry $\left(\AA{ }^{\circ}\right)$.

$\mathrm{Cg} 2$ is the centroid of the $\mathrm{C} 5-\mathrm{C} 10$ ring.

\begin{tabular}{lllll}
\hline$D-\mathrm{H} \cdots A$ & $D-\mathrm{H}$ & $\mathrm{H} \cdots A$ & $D \cdots A$ & $D-\mathrm{H} \cdots A$ \\
\hline $\mathrm{C} 6-\mathrm{H} 6 \cdots \mathrm{O} 1^{\mathrm{i}}$ & 0.95 & 2.46 & $3.406(3)$ & 174 \\
$\mathrm{C} 10-\mathrm{H} 10 \cdots \mathrm{O} 2$ & 0.95 & 2.54 & $2.917(3)$ & 104 \\
$\mathrm{C} 11-\mathrm{H} 11 C \cdots C g 2^{\mathrm{ii}}$ & 0.98 & 2.73 & $3.614(3)$ & 150 \\
\hline
\end{tabular}

Symmetry codes: (i) $-x+1,-y+1,-z$; (ii) $-x,-y+1,-z+1$.

ring are oriented gauche to the $\mathrm{S} 1-\mathrm{C} 5$ bond with torsion angles $\mathrm{C} 5-\mathrm{S} 1-\mathrm{N} 1-\mathrm{C} 1=-65.62(18)^{\circ}$ and $\mathrm{C} 5-\mathrm{S} 1-\mathrm{N} 1-$ $\mathrm{C} 4=76.16(19)^{\circ}$. A conformational analysis of the fivemembered pyrrolidine ring pucker gives a puckering amplitude $\left(Q_{2}\right)$ parameter of $0.352(3) \AA$ and a $\varphi_{2}$ parameter of $262.2(4)^{\circ}$. Consequently, this ring is in a half-chair conformation with a twist along the $\mathrm{C} 2-\mathrm{C} 3$ bond. Lastly, an intramolecular C-H..O contact (Sutor, 1958,1962,1963; Steiner, 1996) is present between $\mathrm{H} 10$ and $\mathrm{O} 2$ with an $\mathrm{H} \cdots A$ distance of $2.54 \AA$ (Table 1).

\section{Supramolecular features}

In the crystal structure of the title compound, molecules are linked by $\pi-\pi$ interactions, $\mathrm{C}-\mathrm{H} \cdots \mathrm{O}$ hydrogen bonds, and $\mathrm{C}-\mathrm{H} \cdots \pi$ interactions (Fig. 3, Table 1). The $\mathrm{C}-\mathrm{H} \cdots \mathrm{O}$ hydrogen bond is formed between an aromatic $\mathrm{C}-\mathrm{H}$ group $(\mathrm{C} 6-\mathrm{H} 6)$ and one of the sulfonamide $\mathrm{O}$ atoms $(\mathrm{O} 1)$. The $\mathrm{C}-$ $\mathrm{H} \cdots \pi$ interaction is between the methyl group $(\mathrm{C} 11-\mathrm{H} 11 C)$ and a symmetry-derived ring (C5-C10; symmetry code: $-x$, $y+1,-z+1)$. The $\pi-\pi$ interaction has a centroid-to-centroid

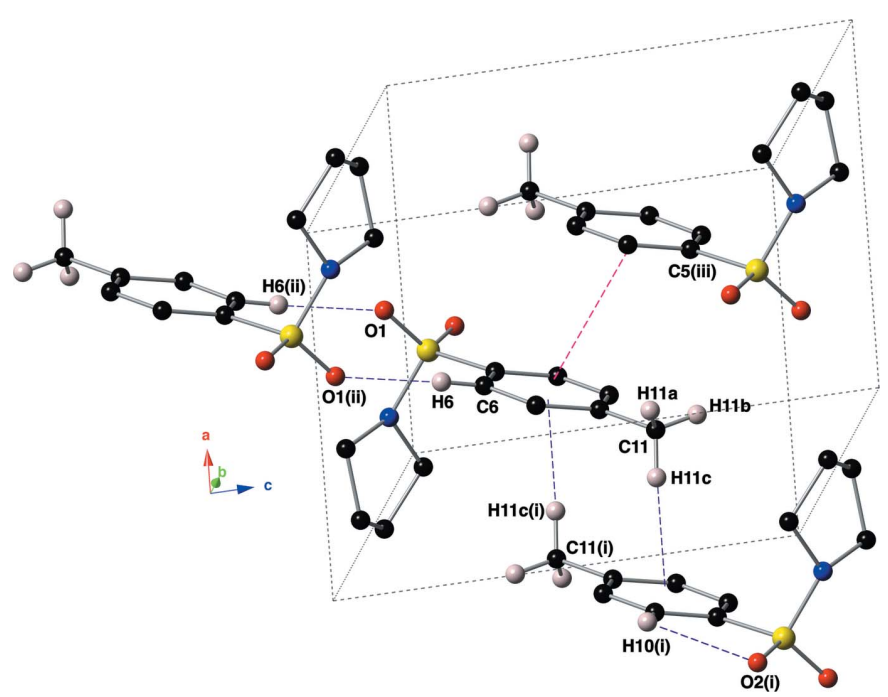

Figure 3

A depiction of the non-covalent interactions present in the crystal of the title compound using a ball-and-stick model with standard CPK colors. $\mathrm{C}-\mathrm{H} \cdots \mathrm{O}$ hydrogen bonds and $\mathrm{C}-\mathrm{H} \cdots \pi$ interactions are shown with purple dashed lines, and $\pi-\pi$ interactions are shown with magenta dashed lines. For clarity, most hydrogen atoms have been omitted and only one orientation of the intramolecular $\mathrm{C}-\mathrm{H} \cdots \mathrm{O}$ hydrogen bond is shown. [Symmetry codes: (i) $-x, 1-y, 1-z$; (ii) $1-x, 1-y,-z$; (iii) $1-x$, $1-y, 1-z$.] 


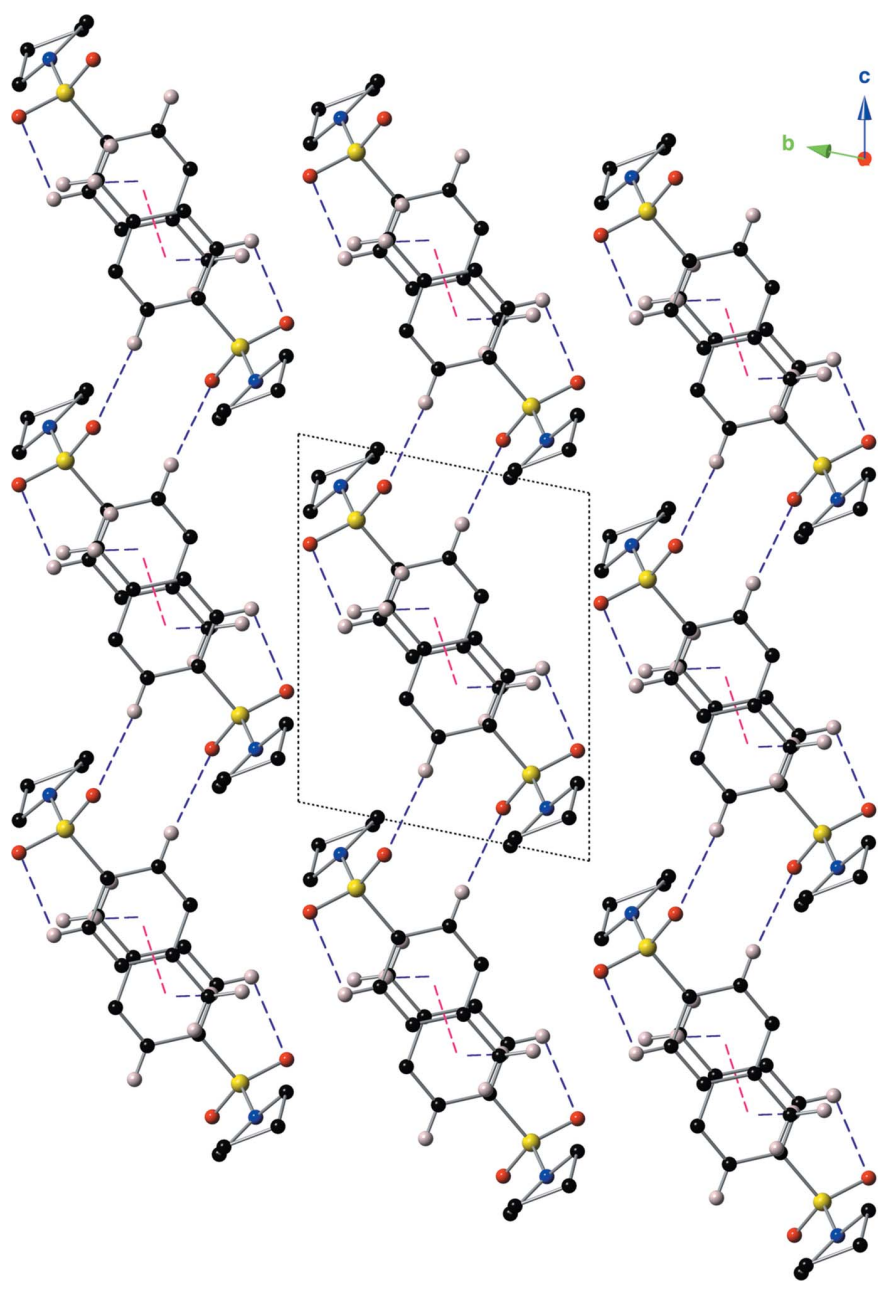

Figure 4

A view down the $a$ axis of the crystal packing showing the supramolecular sheets formed via non-covalent interactions. $\mathrm{C}-\mathrm{H} \cdots \mathrm{O}$ hydrogen bonds and $\mathrm{C}-\mathrm{H} \cdots \pi$ interactions are shown with purple dashed lines, and $\pi-\pi$ interactions are shown with magenta dashed lines. For clarity, only those hydrogen atoms involved in a non-covalent interaction are shown, along with $\mathrm{H} 11 A$ and $\mathrm{H} 11 B$.

distance of 3.8162 (15) A with a slippage of $1.307 \AA$. The result of these interactions is the formation of sheets that lie in the $a c$ plane (Fig. 4).

\section{Database survey}

The Cambridge Structural Database (CSD, Version 5.40, August 2019; Groom et al., 2016) contains hundreds of structures that comprise a $p$-toluenesulfonamide group bearing a pyrrolidine ring. Included in this list is another crystal-structure determination of the title compound (refcode: BABLEV; Ohwada et al., 1998), which also crystallizes in the $P \overline{1}$ space group. Unfortunately, coordinates were not deposited for this structure at that time, so we are unable to say whether the title compound is a new packing polymorph or a new conformational polymorph. The reduced cell of BABLEV is $a=8.241, b$ $=2.671, c=9.240 \AA, \alpha=76.550, \beta=63.800, \gamma=87.880^{\circ}$ with a volume of $574.55 \AA^{3}$. The theoretical X-ray density values for
Table 2

Experimental details.

\begin{tabular}{ll}
\hline Crystal data & \\
Chemical formula & $\mathrm{C}_{11} \mathrm{H}_{15} \mathrm{NO}_{2} \mathrm{~S}$ \\
$M_{\mathrm{r}}$ & 225.30 \\
Crystal system, space group & Triclinic, $P \overline{1}$ \\
Temperature $(\mathrm{K})$ & 173 \\
$a, b, c(\AA)$ & $7.5347(1), 8.2581(1), 9.6157(1)$ \\
$\alpha, \beta, \gamma\left({ }^{\circ}\right)$ & $77.876(1), 86.132(1), 69.682(1)$ \\
$V\left(\AA^{3}\right)$ & $548.56(1)$ \\
$Z$ & 2 \\
Radiation type & $\mathrm{Cu} K \alpha$ \\
$\mu\left(\mathrm{mm}^{-1}\right)$ & 2.46 \\
Crystal size (mm) & $0.22 \times 0.16 \times 0.04$ \\
& \\
Data collection & Bruker APEXII CCD \\
Diffractometer & Multi-scan $(S A D A B S ;$ Krause $e t$ \\
Absorption correction & $a l ., 2015)$ \\
& $0.631,0.753$ \\
$T_{\text {min }}, T_{\text {max }}$ & $7120,1944,1715$ \\
No. of measured, independent and & \\
$\quad$ observed $[I>2 \sigma(I)]$ reflections & 0.033 \\
$R_{\text {int }}$ & 0.603 \\
(sin $\theta / \lambda)_{\text {max }}\left(\AA^{-1}\right)$ & \\
Refinement & \\
$R\left[F^{2}>2 \sigma\left(F^{2}\right)\right], w R\left(F^{2}\right), S$ & $0.041,0.120,1.09$ \\
No. of reflections & 1944 \\
No. of parameters & 137 \\
H-atom treatment & H-atom parameters constrained \\
$\Delta \rho_{\max }, \Delta \rho_{\text {min }}\left(\mathrm{e} \AA^{-3}\right)$ & $0.53,-0.33$ \\
\hline
\end{tabular}

Computer programs: APEX2 and SAINT (Bruker, 2013), SHELXT (Sheldrick, 2015), OLEX2 (Dolomanov et al., 2009; Bourhis et al., 2015) and CrystalMaker (Palmer, 2007).

each structure are similar, with $1.30 \mathrm{~g} \mathrm{~cm}^{-3}$ for BABLEV and $1.36 \mathrm{~g} \mathrm{~cm}^{-3}$ for the title compound. Thus, the more densely packed structure reported here is likely the more thermodynamically stable form.

A selection of other structures in the CSD that are closely related to the title compound are BOKPEX (Rao \& Chan, 2008), GAWDAK (Chen et al., 2005), VECTUT (Sherman et al., 2007) and YIRCOS (Wang \& Peng, 2008). These structures were chosen for comparison because they have relatively simple substituents on the pyrrolidine ring. In their paper describing the structure of GAWDAK, the authors report that this crystal also features both intra- and intermolecular hydrogen bonds in the solid state.

\section{Synthesis and crystallization}

The title compound was prepared by the dropwise addition of $p$-toluenesulfonyl chloride $(1.00 \mathrm{~g}, 5.25 \mathrm{mmol})$ to a stirring mixture of pyrrolidine $(0.48 \mathrm{ml}, 5.90 \mathrm{mmol})$ and $10 \mathrm{ml}$ of tetrahydrofuran. This was followed by the dropwise addition of $0.59 M$ aqueous potassium carbonate $(10 \mathrm{ml}, 5.90 \mathrm{mmol})$ and the mixture was stirred at room temperate for $6 \mathrm{~h}$. Upon acidification with $5 \mathrm{M} \mathrm{HCl}$, a white precipitate was isolated by vacuum filtration to give the crude sulfonamide product. The crude product was dissolved in hot ethanol and filtered. The filtrate was transferred to a scintillation vial and crystallized upon standing for $24 \mathrm{~h}$ to afford colorless crystals, filtered from the mother liquor (yield 91\%; m.p. 405-407 K). 


\section{Refinement}

Crystal data, data collection and structure refinement details are summarized in Table 2. Hydrogen atoms bonded to carbon atoms were placed in calculated positions and refined as riding: $\mathrm{C}-\mathrm{H}=0.95-1.00 \AA$ with $U_{\text {iso }}(\mathrm{H})=1.2 U_{\text {eq }}(\mathrm{C})$ for methylene groups and aromatic hydrogen atoms, and $U_{\text {iso }}(\mathrm{H})$ $=1.5 U_{\text {eq }}(\mathrm{C})$ for methyl groups.

\section{Acknowledgements}

The authors thank Pfizer, Inc. for the donation of a Varian INOVA 400 FT NMR spectrometer. The CCD-based X-ray diffractometers at Michigan State University were upgraded and/or replaced by departmental funds.

\section{Funding information}

Funding for this research was provided by: National Science Foundation (grant No. MRI CHE-1725699); Grand Valley State University Chemistry Department's Weldon Fund.

\section{References}

Apaydın, S. \& Török, M. (2019). Bioorg. Med. Chem. Lett. 29, $2042-$ 2050.

Bourhis, L. J., Dolomanov, O. V., Gildea, R. J., Howard, J. A. K. \& Puschmann, H. (2015). Acta Cryst. A71, 59-75.

Bruker (2013). APEX2, SAINT and SADABS. Bruker AXS Inc. Madison, Wisconsin, USA.
Chen, Y.-J., Xu, G., Cui, Y.-B., Huang, W. \& Gou, S.-H. (2005). Acta Cryst. E61, o3571-o3573.

De Luca, L. \& Giacomelli, G. (2008). J. Org. Chem. 73, 3967-3969.

Dolomanov, O. V., Bourhis, L. J., Gildea, R. J., Howard, J. A. K. \& Puschmann, H. (2009). J. Appl. Cryst. 42, 339-341.

Groom, C. R., Bruno, I. J., Lightfoot, M. P. \& Ward, S. C. (2016). Acta Cryst. B72, 171-179.

Jiang, Y. \& Ma, D. (2013). Top. Organomet. Chem. 46, 87-118.

Krause, L., Herbst-Irmer, R., Sheldrick, G. M. \& Stalke, D. (2015). J. Appl. Cryst. 48, 3-10.

Ohwada, T., Okamoto, I., Shudo, K. \& Yamaguchi, K. (1998). Tetrahedron Lett. 39, 7877-7880.

Palmer, D. (2007). CrystalMaker. CrystalMaker Software, Bicester, England.

Patel, Z. S., Stevens, A. C., Bookout, E. C., Staples, R. J., Biros, S. M. \& Ngassa, F. N. (2018). Acta Cryst. E74, 1126-1129.

Rao, W. \& Chan, W. H. (2008). Chem. Eur. J. 14, 10486-10495.

Sheldrick, G. M. (2015). Acta Cryst. A71, 3-8.

Sherman, E. S., Fuller, P. H., Kasi, D. \& Chemler, S. R. (2007). J. Org. Chem. 72, 3896-3905.

Steiner, T. (1996). Crystallogr. Rev. 6, 1-51.

Sutor, D. J. (1958). Acta Cryst. 11, 453-458.

Sutor, D. J. (1962). Nature, 195, 68-69.

Sutor, D. J. (1963). J. Chem. Soc. pp. 1105-1110.

Ugwu, D. I., Okoro, U. C. \& Ahmad, H. (2017). PLoS One, 12 art. no. e0183807.

Ugwu, D. I., Okoro, U. C. \& Mishra, N. K. (2018). Eur. J. Med. Chem. 154, 110-116.

Wang, Y.-W. \& Peng, Y. (2008). Acta Cryst. E64, o56.

Yan, J., Li, J. \& Cheng, D. (2007). Synlett, pp. 2442-2444.

Yang, L., Powell, D. R. \& Houser, R. P. (2007). Dalton Trans. pp. 955964. 


\title{
supporting information
}

Acta Cryst. (2020). E76, 452-455 [https://doi.org/10.1107/S205698902000208X]

\section{Crystal structure of 1-[(4-methylbenzene)sulfonyl]pyrrolidine}

\author{
Brock A. Stenfors, Richard J. Staples, Shannon M. Biros and Felix N. Ngassa
}

Computing details

Data collection: APEX2 (Bruker, 2013); cell refinement: SAINT (Bruker, 2013); data reduction: SAINT (Bruker, 2013); program(s) used to solve structure: ShelXT (Sheldrick, 2015); program(s) used to refine structure: OLEX2 (Dolomanov et al., 2009; Bourhis et al., 2015); molecular graphics: OLEX2 (Dolomanov et al., 2009; Bourhis et al., 2015); software used to prepare material for publication: CrystalMaker (Palmer, 2007).

1-[(4-Methylbenzene)sulfonyl] pyrrolidine

Crystal data

$\mathrm{C}_{11} \mathrm{H}_{15} \mathrm{NO}_{2} \mathrm{~S}$

$M_{r}=225.30$

Triclinic, $P \overline{1}$

$a=7.5347$ (1) $\AA$

$b=8.2581(1) \AA$

$c=9.6157(1) \AA$

$\alpha=77.876(1)^{\circ}$

$\beta=86.132(1)^{\circ}$

$\gamma=69.682(1)^{\circ}$

$V=548.56(1) \AA^{3}$

Data collection

Bruker APEXII CCD

diffractometer

$\varphi$ and $\omega$ scans

Absorption correction: multi-scan

(SADABS; Krause et al., 2015)

$T_{\min }=0.631, T_{\max }=0.753$

7120 measured reflections

Refinement

Refinement on $F^{2}$

Least-squares matrix: full

$R\left[F^{2}>2 \sigma\left(F^{2}\right)\right]=0.041$

$w R\left(F^{2}\right)=0.120$

$S=1.09$

1944 reflections

137 parameters

0 restraints

Primary atom site location: dual
$Z=2$

$F(000)=240$

$D_{\mathrm{x}}=1.364 \mathrm{Mg} \mathrm{m}^{-3}$

$\mathrm{Cu} K \alpha$ radiation, $\lambda=1.54178 \AA$

Cell parameters from 3988 reflections

$\theta=4.7-68.2^{\circ}$

$\mu=2.46 \mathrm{~mm}^{-1}$

$T=173 \mathrm{~K}$

Plate, colourless

$0.22 \times 0.16 \times 0.04 \mathrm{~mm}$

1944 independent reflections

1715 reflections with $I>2 \sigma(I)$

$R_{\text {int }}=0.033$

$\theta_{\text {max }}=68.3^{\circ}, \theta_{\min }=4.7^{\circ}$

$h=-9 \rightarrow 9$

$k=-9 \rightarrow 9$

$l=-11 \rightarrow 11$

Hydrogen site location: inferred from neighbouring sites

$\mathrm{H}$-atom parameters constrained

$w=1 /\left[\sigma^{2}\left(F_{\mathrm{o}}^{2}\right)+(0.0698 P)^{2}+0.2666 P\right]$

where $P=\left(F_{\mathrm{o}}{ }^{2}+2 F_{\mathrm{c}}{ }^{2}\right) / 3$

$(\Delta / \sigma)_{\max }<0.001$

$\Delta \rho_{\max }=0.53 \mathrm{e} \AA^{-3}$

$\Delta \rho_{\min }=-0.33$ e $\AA^{-3}$ 


\section{Special details}

Geometry. All esds (except the esd in the dihedral angle between two 1.s. planes) are estimated using the full covariance matrix. The cell esds are taken into account individually in the estimation of esds in distances, angles and torsion angles; correlations between esds in cell parameters are only used when they are defined by crystal symmetry. An approximate (isotropic) treatment of cell esds is used for estimating esds involving l.s. planes.

Fractional atomic coordinates and isotropic or equivalent isotropic displacement parameters $\left(\AA^{2}\right)$

\begin{tabular}{lllll}
\hline & $x$ & $y$ & $z$ & $U_{\text {iso }} * / U_{\text {eq }}$ \\
\hline S1 & $0.56522(7)$ & $0.19952(7)$ & $0.20258(5)$ & $0.0225(2)$ \\
O1 & $0.6545(2)$ & $0.2957(2)$ & $0.09657(16)$ & $0.0302(4)$ \\
O2 & $0.6789(2)$ & $0.0403(2)$ & $0.29321(17)$ & $0.0313(4)$ \\
N1 & $0.4193(3)$ & $0.1465(2)$ & $0.11936(19)$ & $0.0239(4)$ \\
C1 & $0.3110(3)$ & $0.0415(3)$ & $0.2046(2)$ & $0.0283(5)$ \\
H1A & 0.263738 & 0.084458 & 0.293341 & $0.034^{*}$ \\
H1B & 0.389620 & -0.084784 & 0.228939 & $0.034^{*}$ \\
C2 & $0.1485(3)$ & $0.0702(3)$ & $0.1065(3)$ & $0.0353(6)$ \\
H2A & 0.184331 & -0.016766 & 0.043620 & $0.042^{*}$ \\
H2B & 0.035011 & 0.061924 & 0.161429 & $0.042^{*}$ \\
C3 & $0.1137(4)$ & $0.2554(4)$ & $0.0214(4)$ & $0.0511(8)$ \\
H3A & 0.051601 & 0.272209 & -0.070616 & $0.061^{*}$ \\
H3B & 0.032278 & 0.344031 & 0.074899 & $0.061^{*}$ \\
C4 & $0.3053(4)$ & $0.2719(3)$ & $-0.0018(3)$ & $0.0357(6)$ \\
H4A & 0.359019 & 0.240275 & -0.093216 & $0.043^{*}$ \\
H4B & 0.299424 & 0.393584 & -0.001822 & $0.043^{*}$ \\
C5 & $0.4298(3)$ & $0.3438(3)$ & $0.3132(2)$ & $0.0225(5)$ \\
C6 & $0.3318(3)$ & $0.5190(3)$ & $0.2538(2)$ & $0.0287(5)$ \\
H6 & 0.342164 & 0.563001 & 0.155156 & $0.034^{*}$ \\
C7 & $0.2192(3)$ & $0.6284(3)$ & $0.3400(3)$ & $0.0331(6)$ \\
H7 & 0.149316 & 0.747265 & 0.299159 & $0.040^{*}$ \\
C8 & $0.2063(3)$ & $0.5674(4)$ & $0.4859(3)$ & $0.0334(6)$ \\
C9 & $0.3052(4)$ & $0.3933(4)$ & $0.5423(3)$ & $0.0359(6)$ \\
H9 & 0.296907 & 0.349698 & 0.641258 & $0.043^{*}$ \\
C10 & $0.4167(3)$ & $0.2805(3)$ & $0.4575(2)$ & $0.0296(5)$ \\
H10 & 0.483503 & 0.160696 & 0.497955 & $0.035^{*}$ \\
C11 & $0.0852(4)$ & $0.6900(4)$ & $0.5788(3)$ & $0.0516(8)$ \\
H11A & 0.096515 & 0.806829 & 0.547317 & $0.077^{*}$ \\
H11B & 0.127699 & 0.643344 & 0.677852 & $0.077^{*}$ \\
H11C & -0.047268 & 0.699432 & 0.571301 & $0.077^{*}$ \\
& & & &
\end{tabular}

Atomic displacement parameters $\left(\AA^{2}\right)$

\begin{tabular}{lllllll}
\hline & $U^{11}$ & $U^{22}$ & $U^{33}$ & $U^{12}$ & $U^{13}$ & $U^{23}$ \\
\hline S1 & $0.0196(3)$ & $0.0255(3)$ & $0.0225(3)$ & $-0.0078(2)$ & $-0.00028(19)$ & $-0.0042(2)$ \\
O1 & $0.0262(8)$ & $0.0370(10)$ & $0.0289(9)$ & $-0.0143(7)$ & $0.0064(6)$ & $-0.0056(7)$ \\
O2 & $0.0243(8)$ & $0.0313(9)$ & $0.0345(9)$ & $-0.0062(7)$ & $-0.0048(7)$ & $-0.0023(7)$ \\
N1 & $0.0240(9)$ & $0.0269(10)$ & $0.0223(9)$ & $-0.0101(8)$ & $-0.0003(7)$ & $-0.0057(7)$ \\
C1 & $0.0278(11)$ & $0.0262(12)$ & $0.0327(12)$ & $-0.0117(9)$ & $-0.0001(9)$ & $-0.0051(9)$
\end{tabular}




\begin{tabular}{lllllll} 
C2 & $0.0280(12)$ & $0.0392(15)$ & $0.0423(15)$ & $-0.0142(10)$ & $-0.0049(10)$ & $-0.0095(11)$ \\
C3 & $0.0361(15)$ & $0.0509(18)$ & $0.0596(19)$ & $-0.0150(13)$ & $-0.0176(13)$ & $0.0090(14)$ \\
C4 & $0.0395(14)$ & $0.0379(14)$ & $0.0281(13)$ & $-0.0140(11)$ & $-0.0081(10)$ & $0.0005(10)$ \\
C5 & $0.0217(10)$ & $0.0269(12)$ & $0.0224(11)$ & $-0.0113(9)$ & $-0.0004(8)$ & $-0.0069(8)$ \\
C6 & $0.0318(12)$ & $0.0295(13)$ & $0.0267(12)$ & $-0.0141(10)$ & $0.0011(9)$ & $-0.0039(9)$ \\
C7 & $0.0316(12)$ & $0.0262(13)$ & $0.0443(15)$ & $-0.0114(10)$ & $0.0041(10)$ & $-0.0118(10)$ \\
C8 & $0.0288(12)$ & $0.0453(15)$ & $0.0383(14)$ & $-0.0212(11)$ & $0.0072(10)$ & $-0.0215(11)$ \\
C9 & $0.0370(13)$ & $0.0553(17)$ & $0.0224(12)$ & $-0.0224(12)$ & $0.0024(10)$ & $-0.0119(11)$ \\
C10 & $0.0302(12)$ & $0.0361(14)$ & $0.0225(11)$ & $-0.0122(10)$ & $-0.0032(9)$ & $-0.0035(9)$ \\
C11 & $0.0418(15)$ & $0.070(2)$ & $0.0624(19)$ & $-0.0267(15)$ & $0.0161(14)$ & $-0.0471(17)$ \\
\hline
\end{tabular}

Geometric parameters $\left(\hat{A},{ }^{o}\right)$

\begin{tabular}{|c|c|c|c|}
\hline $\mathrm{S} 1-\mathrm{O} 1$ & $1.4357(16)$ & $\mathrm{C} 4-\mathrm{H} 4 \mathrm{~B}$ & 0.9900 \\
\hline $\mathrm{S} 1-\mathrm{O} 2$ & $1.4349(16)$ & $\mathrm{C} 5-\mathrm{C} 6$ & $1.390(3)$ \\
\hline $\mathrm{S} 1-\mathrm{N} 1$ & $1.6248(18)$ & $\mathrm{C} 5-\mathrm{C} 10$ & $1.386(3)$ \\
\hline $\mathrm{S} 1-\mathrm{C} 5$ & $1.770(2)$ & C6- $-\mathrm{H} 6$ & 0.9500 \\
\hline $\mathrm{N} 1-\mathrm{C} 1$ & $1.481(3)$ & $\mathrm{C} 6-\mathrm{C} 7$ & $1.382(3)$ \\
\hline $\mathrm{N} 1-\mathrm{C} 4$ & $1.476(3)$ & $\mathrm{C} 7-\mathrm{H} 7$ & 0.9500 \\
\hline $\mathrm{C} 1-\mathrm{H} 1 \mathrm{~A}$ & 0.9900 & $\mathrm{C} 7-\mathrm{C} 8$ & $1.397(4)$ \\
\hline $\mathrm{C} 1-\mathrm{H} 1 \mathrm{~B}$ & 0.9900 & $\mathrm{C} 8-\mathrm{C} 9$ & $1.379(4)$ \\
\hline $\mathrm{C} 1-\mathrm{C} 2$ & $1.518(3)$ & $\mathrm{C} 8-\mathrm{C} 11$ & $1.511(3)$ \\
\hline $\mathrm{C} 2-\mathrm{H} 2 \mathrm{~A}$ & 0.9900 & C9-H9 & 0.9500 \\
\hline $\mathrm{C} 2-\mathrm{H} 2 \mathrm{~B}$ & 0.9900 & $\mathrm{C} 9-\mathrm{C} 10$ & $1.386(3)$ \\
\hline $\mathrm{C} 2-\mathrm{C} 3$ & $1.517(4)$ & $\mathrm{C} 10-\mathrm{H} 10$ & 0.9500 \\
\hline $\mathrm{C} 3-\mathrm{H} 3 \mathrm{~A}$ & 0.9900 & $\mathrm{C} 11-\mathrm{H} 11 \mathrm{~A}$ & 0.9800 \\
\hline $\mathrm{C} 3-\mathrm{H} 3 \mathrm{~B}$ & 0.9900 & $\mathrm{C} 11-\mathrm{H} 11 \mathrm{~B}$ & 0.9800 \\
\hline $\mathrm{C} 3-\mathrm{C} 4$ & $1.495(4)$ & $\mathrm{C} 11-\mathrm{H} 11 \mathrm{C}$ & 0.9800 \\
\hline $\mathrm{C} 4-\mathrm{H} 4 \mathrm{~A}$ & 0.9900 & & \\
\hline $\mathrm{O} 1-\mathrm{S} 1-\mathrm{N} 1$ & $106.88(9)$ & $\mathrm{N} 1-\mathrm{C} 4-\mathrm{H} 4 \mathrm{~B}$ & 110.9 \\
\hline $\mathrm{O} 1-\mathrm{S} 1-\mathrm{C} 5$ & $108.04(10)$ & $\mathrm{C} 3-\mathrm{C} 4-\mathrm{H} 4 \mathrm{~A}$ & 110.9 \\
\hline $\mathrm{O} 2-\mathrm{S} 1-\mathrm{O} 1$ & $119.67(10)$ & $\mathrm{C} 3-\mathrm{C} 4-\mathrm{H} 4 \mathrm{~B}$ & 110.9 \\
\hline $\mathrm{O} 2-\mathrm{S} 1-\mathrm{N} 1$ & $106.48(10)$ & $\mathrm{H} 4 \mathrm{~A}-\mathrm{C} 4-\mathrm{H} 4 \mathrm{~B}$ & 108.9 \\
\hline $\mathrm{O} 2-\mathrm{S} 1-\mathrm{C} 5$ & $107.59(10)$ & $\mathrm{C} 6-\mathrm{C} 5-\mathrm{S} 1$ & $119.68(17)$ \\
\hline $\mathrm{N} 1-\mathrm{S} 1-\mathrm{C} 5$ & $107.66(9)$ & $\mathrm{C} 10-\mathrm{C} 5-\mathrm{S} 1$ & $120.00(18)$ \\
\hline $\mathrm{C} 1-\mathrm{N} 1-\mathrm{S} 1$ & $118.02(14)$ & $\mathrm{C} 10-\mathrm{C} 5-\mathrm{C} 6$ & $120.3(2)$ \\
\hline $\mathrm{C} 4-\mathrm{N} 1-\mathrm{S} 1$ & $120.69(16)$ & $\mathrm{C} 5-\mathrm{C} 6-\mathrm{H} 6$ & 120.4 \\
\hline $\mathrm{C} 4-\mathrm{N} 1-\mathrm{C} 1$ & $110.89(17)$ & $\mathrm{C} 7-\mathrm{C} 6-\mathrm{C} 5$ & $119.2(2)$ \\
\hline $\mathrm{N} 1-\mathrm{C} 1-\mathrm{H} 1 \mathrm{~A}$ & 111.1 & $\mathrm{C} 7-\mathrm{C} 6-\mathrm{H} 6$ & 120.4 \\
\hline $\mathrm{N} 1-\mathrm{C} 1-\mathrm{H} 1 \mathrm{~B}$ & 111.1 & $\mathrm{C} 6-\mathrm{C} 7-\mathrm{H} 7$ & 119.4 \\
\hline $\mathrm{N} 1-\mathrm{C} 1-\mathrm{C} 2$ & $103.35(18)$ & $\mathrm{C} 6-\mathrm{C} 7-\mathrm{C} 8$ & $121.3(2)$ \\
\hline $\mathrm{H} 1 \mathrm{~A}-\mathrm{C} 1-\mathrm{H} 1 \mathrm{~B}$ & 109.1 & $\mathrm{C} 8-\mathrm{C} 7-\mathrm{H} 7$ & 119.4 \\
\hline $\mathrm{C} 2-\mathrm{C} 1-\mathrm{H} 1 \mathrm{~A}$ & 111.1 & $\mathrm{C} 7-\mathrm{C} 8-\mathrm{C} 11$ & $120.4(3)$ \\
\hline $\mathrm{C} 2-\mathrm{C} 1-\mathrm{H} 1 \mathrm{~B}$ & 111.1 & $\mathrm{C} 9-\mathrm{C} 8-\mathrm{C} 7$ & $118.4(2)$ \\
\hline $\mathrm{C} 1-\mathrm{C} 2-\mathrm{H} 2 \mathrm{~A}$ & 111.1 & $\mathrm{C} 9-\mathrm{C} 8-\mathrm{C} 11$ & $121.2(3)$ \\
\hline $\mathrm{C} 1-\mathrm{C} 2-\mathrm{H} 2 \mathrm{~B}$ & 111.1 & $\mathrm{C} 8-\mathrm{C} 9-\mathrm{H} 9$ & 119.3 \\
\hline $\mathrm{H} 2 \mathrm{~A}-\mathrm{C} 2-\mathrm{H} 2 \mathrm{~B}$ & 109.1 & $\mathrm{C} 8-\mathrm{C} 9-\mathrm{C} 10$ & $121.3(2)$ \\
\hline
\end{tabular}




\begin{tabular}{|c|c|c|c|}
\hline $\mathrm{C} 3-\mathrm{C} 2-\mathrm{C} 1$ & $103.2(2)$ & $\mathrm{C} 10-\mathrm{C} 9-\mathrm{H} 9$ & 119.3 \\
\hline $\mathrm{C} 3-\mathrm{C} 2-\mathrm{H} 2 \mathrm{~A}$ & 111.1 & $\mathrm{C} 5-\mathrm{C} 10-\mathrm{C} 9$ & $119.5(2)$ \\
\hline $\mathrm{C} 3-\mathrm{C} 2-\mathrm{H} 2 \mathrm{~B}$ & 111.1 & $\mathrm{C} 5-\mathrm{C} 10-\mathrm{H} 10$ & 120.2 \\
\hline $\mathrm{C} 2-\mathrm{C} 3-\mathrm{H} 3 \mathrm{~A}$ & 110.7 & $\mathrm{C} 9-\mathrm{C} 10-\mathrm{H} 10$ & 120.2 \\
\hline $\mathrm{C} 2-\mathrm{C} 3-\mathrm{H} 3 \mathrm{~B}$ & 110.7 & $\mathrm{C} 8-\mathrm{C} 11-\mathrm{H} 11 \mathrm{~A}$ & 109.5 \\
\hline $\mathrm{H} 3 \mathrm{~A}-\mathrm{C} 3-\mathrm{H} 3 \mathrm{~B}$ & 108.8 & $\mathrm{C} 8-\mathrm{C} 11-\mathrm{H} 11 \mathrm{~B}$ & 109.5 \\
\hline $\mathrm{C} 4-\mathrm{C} 3-\mathrm{C} 2$ & $105.2(2)$ & $\mathrm{C} 8-\mathrm{C} 11-\mathrm{H} 11 \mathrm{C}$ & 109.5 \\
\hline $\mathrm{C} 4-\mathrm{C} 3-\mathrm{H} 3 \mathrm{~A}$ & 110.7 & $\mathrm{H} 11 \mathrm{~A}-\mathrm{C} 11-\mathrm{H} 11 \mathrm{~B}$ & 109.5 \\
\hline $\mathrm{C} 4-\mathrm{C} 3-\mathrm{H} 3 \mathrm{~B}$ & 110.7 & $\mathrm{H} 11 \mathrm{~A}-\mathrm{C} 11-\mathrm{H} 11 \mathrm{C}$ & 109.5 \\
\hline $\mathrm{N} 1-\mathrm{C} 4-\mathrm{C} 3$ & $104.3(2)$ & $\mathrm{H} 11 \mathrm{~B}-\mathrm{C} 11-\mathrm{H} 11 \mathrm{C}$ & 109.5 \\
\hline $\mathrm{N} 1-\mathrm{C} 4-\mathrm{H} 4 \mathrm{~A}$ & 110.9 & & \\
\hline $\mathrm{S} 1-\mathrm{N} 1-\mathrm{C} 1-\mathrm{C} 2$ & $161.32(16)$ & $\mathrm{C} 1-\mathrm{N} 1-\mathrm{C} 4-\mathrm{C} 3$ & $6.5(3)$ \\
\hline $\mathrm{S} 1-\mathrm{N} 1-\mathrm{C} 4-\mathrm{C} 3$ & $-137.8(2)$ & $\mathrm{C} 1-\mathrm{C} 2-\mathrm{C} 3-\mathrm{C} 4$ & $36.6(3)$ \\
\hline $\mathrm{S} 1-\mathrm{C} 5-\mathrm{C} 6-\mathrm{C} 7$ & $176.88(17)$ & $\mathrm{C} 2-\mathrm{C} 3-\mathrm{C} 4-\mathrm{N} 1$ & $-26.6(3)$ \\
\hline $\mathrm{S} 1-\mathrm{C} 5-\mathrm{C} 10-\mathrm{C} 9$ & $-177.89(17)$ & $\mathrm{C} 4-\mathrm{N} 1-\mathrm{C} 1-\mathrm{C} 2$ & $16.0(2)$ \\
\hline $\mathrm{O} 1-\mathrm{S} 1-\mathrm{N} 1-\mathrm{C} 1$ & $178.52(15)$ & $\mathrm{C} 5-\mathrm{S} 1-\mathrm{N} 1-\mathrm{C} 1$ & $-65.62(18)$ \\
\hline $\mathrm{O} 1-\mathrm{S} 1-\mathrm{N} 1-\mathrm{C} 4$ & $-39.71(19)$ & $\mathrm{C} 5-\mathrm{S} 1-\mathrm{N} 1-\mathrm{C} 4$ & $76.16(19)$ \\
\hline $\mathrm{O} 1-\mathrm{S} 1-\mathrm{C} 5-\mathrm{C} 6$ & $38.27(19)$ & $\mathrm{C} 5-\mathrm{C} 6-\mathrm{C} 7-\mathrm{C} 8$ & $1.8(3)$ \\
\hline $\mathrm{O} 1-\mathrm{S} 1-\mathrm{C} 5-\mathrm{C} 10$ & $-143.93(18)$ & $\mathrm{C} 6-\mathrm{C} 5-\mathrm{C} 10-\mathrm{C} 9$ & $-0.1(3)$ \\
\hline $\mathrm{O} 2-\mathrm{S} 1-\mathrm{N} 1-\mathrm{C} 1$ & $49.53(18)$ & $\mathrm{C} 6-\mathrm{C} 7-\mathrm{C} 8-\mathrm{C} 9$ & $-1.6(3)$ \\
\hline $\mathrm{O} 2-\mathrm{S} 1-\mathrm{N} 1-\mathrm{C} 4$ & $-168.70(17)$ & $\mathrm{C} 6-\mathrm{C} 7-\mathrm{C} 8-\mathrm{C} 11$ & $178.9(2)$ \\
\hline $\mathrm{O} 2-\mathrm{S} 1-\mathrm{C} 5-\mathrm{C} 6$ & $168.76(16)$ & $\mathrm{C} 7-\mathrm{C} 8-\mathrm{C} 9-\mathrm{C} 10$ & $0.5(3)$ \\
\hline $\mathrm{O} 2-\mathrm{S} 1-\mathrm{C} 5-\mathrm{C} 10$ & $-13.4(2)$ & $\mathrm{C} 8-\mathrm{C} 9-\mathrm{C} 10-\mathrm{C} 5$ & $0.3(3)$ \\
\hline $\mathrm{N} 1-\mathrm{S} 1-\mathrm{C} 5-\mathrm{C} 6$ & $-76.84(19)$ & $\mathrm{C} 10-\mathrm{C} 5-\mathrm{C} 6-\mathrm{C} 7$ & $-0.9(3)$ \\
\hline $\mathrm{N} 1-\mathrm{S} 1-\mathrm{C} 5-\mathrm{C} 10$ & $100.97(19)$ & $\mathrm{C} 11-\mathrm{C} 8-\mathrm{C} 9-\mathrm{C} 10$ & $-179.9(2)$ \\
\hline $\mathrm{N} 1-\mathrm{C} 1-\mathrm{C} 2-\mathrm{C} 3$ & $-31.6(3)$ & & \\
\hline
\end{tabular}

Hydrogen-bond geometry $\left(A,{ }^{\circ}\right)$

$C g 2$ is the centroid of the $\mathrm{C} 5-\mathrm{C} 10$ ring.

\begin{tabular}{lllll}
\hline$D-\mathrm{H} \cdots A$ & $D-\mathrm{H}$ & $\mathrm{H} \cdots A$ & $D \cdots A$ & $D-\mathrm{H} \cdots A$ \\
\hline $\mathrm{C} 6-\mathrm{H} 6 \cdots \mathrm{O} 1^{\mathrm{i}}$ & 0.95 & 2.46 & $3.406(3)$ & 174 \\
$\mathrm{C} 10-\mathrm{H} 10 \cdots \mathrm{O} 2$ & 0.95 & 2.54 & $2.917(3)$ & 104 \\
$\mathrm{C} 11-\mathrm{H} 11 C \cdots C g 2^{\mathrm{ii}}$ & 0.98 & 2.73 & $3.614(3)$ & 150 \\
\hline
\end{tabular}

Symmetry codes: (i) $-x+1,-y+1,-z$; (ii) $-x,-y+1,-z+1$. 